\title{
Latarjet artroscópico: Descrição de técnica cirúrgica e resultados preliminares. Estudo de 30 primeiros casos*
}

\section{Arthroscopic Latarjet: Technique and Preliminary Results. Study from the First 30 Cases}

\author{
Wagner Castropil ${ }^{1}$ Breno Schor ${ }^{1}$ Alexandre Bitar $^{1}$ Giovanna Medina ${ }^{1}$ Luiz Henrique Ribas ${ }^{1}$ \\ Carlos Mendes ${ }^{1}$ \\ ${ }^{1}$ Departamento de Ortopedia, Instituto Vita, São Paulo, SP, Brasil \\ Endereço para correspondência Wagner Castropil, MD, PhD, Instituto \\ Vita, Rua Mato Grosso, 306, $1^{\circ}$ andar, Higienópolis, \\ Rev Bras Ortop 2020;55(2):208-214. \\ São Paulo, SP, 01239-040, Brasil (e-mail: castropil@institutovita.com.br).
}

\section{Resumo \\ Palavras-chave \\ - ombro \\ - luxação do ombro \\ - artroscopia \\ - procedimentos cirúrgicos minimamente invasivos}

Objetivo A realização da cirurgia de Latarjet por via artroscópica tem sido possível pelo desenvolvimento de instrumentais adequados e um protocolo para cirurgia estabelecido e reprodutível, que promete uma técnica mais precisa e com menor incidência de complicações. O objetivo do presente estudo foi fazer uma breve descrição de técnica cirúrgica e avaliar as complicações de curto prazo após a cirurgia de Latarjet por via artroscópica para correção de luxação anterior do ombro com perda óssea glenoidal.

Método Estudo retrospectivo com 30 pacientes com instabilidade anterior do ombro, operados com a técnica de Latarjet por via artroscópica. Foram feitas avaliação das complicações intraoperatórias e pós-operatório de curto prazo e documentação de necessidade de reintervenções.

Resultados Cinco casos tiveram complicação (16,7\%), sendo que nos últimos 10 casos nenhuma complicação foi observada. Em 1 caso (3,3\%) foi necessária conversão para cirurgia aberta por fratura do processo coracoide no momento de fixação na glenoide. Nenhum outro caso apresentou complicação intraoperatória. Não houve infecção nos casos operados. Dois casos (6,7\%) evoluíram com neuropraxia temporária do musculocutâneo, revertida com fisioterapia. Em tempo de seguimento de 6 a 26 meses, 2 pacientes $(6,7 \%)$ necessitaram de nova intervenção para retirada do material de síntese e liberação articular por excessiva limitação da rotação externa. Não houve caso de recidiva.

Conclusão O procedimento de Latarjet artroscópico se mostrou seguro e com baixa incidência de complicações de curto prazo para correção da luxação anterior no ombro com perda óssea na glenoide mesmo em curva inicial de aprendizagem.

\section{Abstract}

Objective Arthroscopic Latarjet has been performed with the aim to be an accurate technique with a low incidence of complications. The aim of the present study was to briefly describe the technique and to evaluate the shot-term complications following

* Trabalho desenvolvido no Instituto Vita, São Paulo, SP, Brasil.

recebido

18 de Agosto de 2018 aceito 22 de Janeiro de 2019
DOI https://doi.org/

10.1055/s-0039-3400523. ISSN $0102-3616$.
Copyright $(2020$ by Sociedade Brasileira License terms de Ortopedia e Traumatologia. Published by Thieme Revinter Publicações Ltda, Rio de Janeiro, Brazil 


\section{Keywords}

- shoulder

- shoulder instability

- arthroscopy

- minimally invasive surgical procedures arthroscopic Latarjet procedure to correct anterior shoulder dislocation with glenoid bone loss.

Methods Retrospective study with 30 subjects with anterior shoulder instability, submitted to arthroscopic Latarjet. Intraoperative and short-term postoperative complications were recorded, as well as the rate of revision surgery.

Results Five cases had complication (16.7\%), and in the last 10 cases no complication occurred. In 1 case (3.3\%), it was required to reverse for open surgery due to a fracture of the coracoid process during fixation in the glenoid. No other intraoperative complication occurred. No infection was observed. Two cases (6.7\%) evolved with temporary neuropraxia of the musculocutaneous nerve, totally reversed with physiotherapy. With a follow-up from 6 to 26 months, 2 patients (6.7\%) required a new intervention for graft/screws removal and release of the joint due to excessive limitation in external rotation. There was no case of recurrence.

Conclusion Even in an initial learning curve, arthroscopic Latarjet demonstrated a low rate of short-tem complications and was a safe procedure for treating anterior dislocation of the shoulder with glenoid bone loss.

\section{Introdução}

A instabilidade anterior é a mais frequente das instabilidades do ombro, sendo uma lesão frequente e potencialmente incapacitante. $\mathrm{O}$ tipo de tratamento nas situações primárias (agudas) e a escolha da técnica cirúrgica são motivo de controvérsia, mas o entendimento que as falhas ósseas na borda anterior da glenoide são a causa principal da recorrência da instabilidade fazem surgir procedimentos para reconstrução óssea. ${ }^{1}$

A evolução do tratamento destas condições começa com Bankart, ${ }^{2}$ que foi quem descreveu em 1923 a lesão essencial na instabilidade anterior do ombro e seu reparo com suturas do lábio e do ligamento glenoumeral inferior na borda anterior da glenoide. Em 1954, Latarjet $^{3}$ descreve técnica para tratamento da luxação anterior do ombro realizando a transferência do processo coracoide para a borda anterior da glenoide e fixando-o com parafuso (-Figura 1). Posteriormente em 1958, Helfet ${ }^{4}$ descreve procedimento semelhante, porém a ponta do processo coracoide era suturada em partes moles junto à borda anterior da glenoide. Ele denomina este procedimento como técnica de Bristow em homenagem ao seu professor. Com a popularização do método, este passou a ser chamado de técnica de Bristow-Latarjet. Em nosso meio,
Ferreira Filho ${ }^{5}$ teve papel fundamental com a padronização e divulgação desta técnica.

A cirurgia de Bristow-Latarjet possibilita dois tipos de efeito na estabilização do ombro ${ }^{6}$ : estático pelo enxerto anterior aumentando a superfície da glenoide e o dinâmico ("sling"), pela ação do tendão conjunto e também por impedir a ascensão do $1 / 3$ inferior do tendão do subescapular. ${ }^{7} \mathrm{~A}$ técnica, apesar de bastante eficaz na estabilização da articulação, apresenta um potencial de complicações significativo: neuropraxias do nervo musculocutâneo e do nervo axilar, problemas relacionados ao enxerto e ao material de síntese e limitação da rotação externa levando à artrose são algumas das complicações que os pacientes podem enfrentar. ${ }^{8}$

Com o desenvolvimento dos instrumentais artroscópicos, o mesmo reparo de Bankart e a mesma capsuloplastia puderam ser feitos por meio de uma cirurgia menos invasiva. A estabilização artroscópica oferecia inúmeras vantagens em relação à estabilização aberta: uma visão $360^{\circ}$ da articulação, menos complicações relacionadas à abertura do subescapular, menor perda sanguínea, menor uso de medicamentos no pós-operatório, além de uma reabilitação mais rápida, manutenção de amplitude de movimentos e estética. ${ }^{8}$ Porém, os resultados iniciais de médio e longo prazo mostravam uma alta taxa de recidiva, de $\sim 15$ a $20 \%$. Com o desenvolvimento de instrumentais e âncoras, esta taxa passou a se assemelhar com a cirurgia
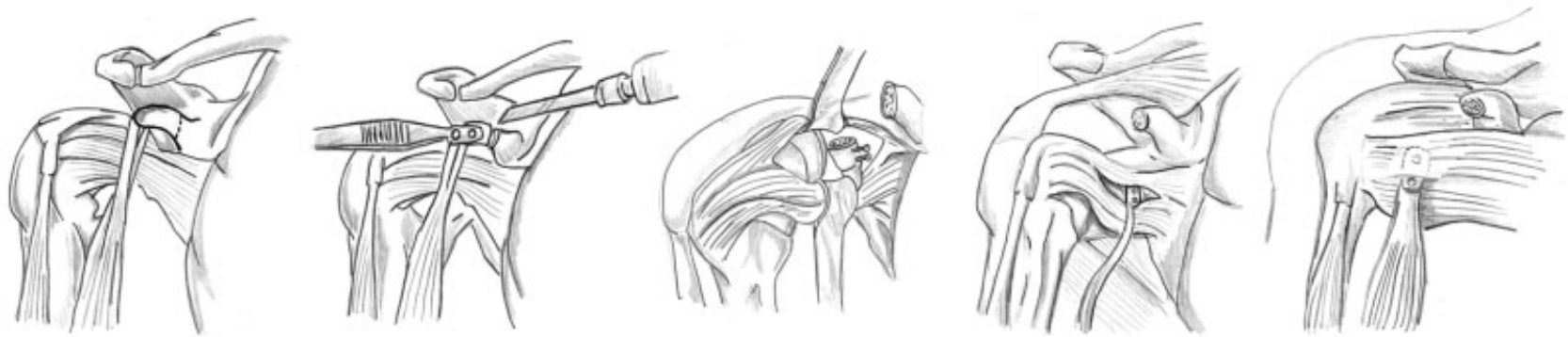

Fig. 1 Ilustração do procedimento de Latarjet. Trajeto e subsequente osteotomia do processo coracoide, alinhamento e subsequente passagem dos dois parafusos e posicionamento final na borda anterior da glenoide. 
aberta, sendo uma escolha do cirurgião a realização deste procedimento por via aberta ou artroscópica. ${ }^{9}$

Em 2007, foi descrita a realização da técnica de Latarjet $^{10}$ por via artroscópica para casos de falha óssea, insuficiência ligamentar, lesão do tipo avulsão umeral do ligamento glenoumeral (AULG) ou falha de reparo de Bankart. Em 2010, foram publicados resultados de 41 casos sem complicações neurológicas ou recidivas, e em 2014 um estudo demonstrou baixa taxa de recidivas em 62 pacientes com seguimento de 5 anos. ${ }^{11,12}$ A partir daí, relatos começaram a ser publicados com grupos ao redor do mundo, mostrando a experiência norteamericana, ${ }^{13}$ chinesa, $^{14}$ italiana $^{15}$ e alemã, ${ }^{16}$ reproduzindo a técnica com segurança, sendo tema de recente editorial da revista Arthroscopy no final de 2017. ${ }^{17}$

O presente estudo objetivou avaliar os resultados de curto prazo e complicações precoces da cirurgia para correção de luxação anterior no ombro com perda óssea na glenoide pela técnica de Latarjet por via artroscópica em experiência inicial de um único centro.

\section{Materiais e Métodos}

Este é um estudo retrospectivo, de série de casos realizado em um único centro. Os participantes analisados no presente estudo realizaram o procedimento cirúrgico em questão entre 2015 e 2017, e foram seguidos por no mínimo 6 meses após o procedimento. $\mathrm{O}$ referido estudo foi aprovado pelo Comitê de Ética em Pesquisa com Seres Humanos, sob o CAAE 88372518.2.0000.5485.

Foram incluídos no presente estudo pacientes com os seguintes critérios: com defeitos ósseos na borda anterior da glenoide $>20 \%$ ou "engaging" Hill-Sachs (avaliados por meio de ressonância magnética [RM] ou tomografia computadorizada [TC]) e questionário Instability Severity Index Score (ISIS) $)^{18}$ $>6$ ou casos de falha no reparo de Bankart; casos submetidos à cirurgia de Latarjet por via artroscópica; mínimo de 6 meses de seguimento. Pacientes com lesão do manguito rotador ou com fraturas do terço proximal do úmero não foram incluídos. No total foram incluídos 30 pacientes.

No presente trabalho, foram avaliadas intercorrências relacionadas ao procedimento. Além disso, foram coletadas as complicações precoces do procedimento, assim como a necessidade de nova intervenção cirúrgica. Os dados foram coletados a partir de prontuário médico. Não houve cálculo amostral por se tratar de estudo de grupo único e não comparativo.

As informações que foram coletadas segundo os desfechos descritos foram apresentadas através de estatística descritiva para descrever e resumir o conjunto de dados.

\section{Técnica Cirúrgica}

Os pacientes foram operados na posição em cadeira de praia, com uma leve inclinação da cabeça para o lado contralateral e colocação dos campos que permitam uma exposição do tórax mais medial. $O$ posicionamento do portal posterior, em linha com a articulação, é fundamental para o adequado posicionamento do portal $\mathrm{M}$ e o correto ângulo para fixação do enxerto na glenoide. Os portais utilizados foram os descritos por Lafosse e estão ilustrados na - Figura 2.

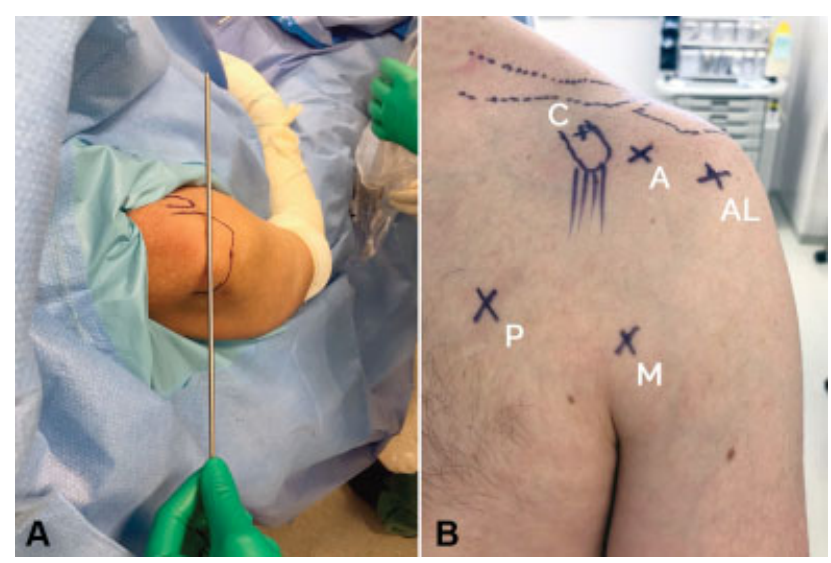

Fig. 2 Portais artroscópicos para realização do Latarjet artroscópico. Figura A. Posicionamento do portal posterior (em linha com a articulação). Figura B. Portais mostrados na imagem em sentido horário: (M) mid-sub; $(P)$ peitoral; $(C)$ coracoide; $(A)$ anterior; $(A L)$ ântero-lateral.

A cirurgia pode ser descrita em etapas:

1. Identificação da lesão de Bankart e abertura do espaço rotador (visualização: portal posterior; portal de trabalho: anterior)

A cirurgia se inicia com o portal posterior com o detalhe que o mesmo deve ser posicionado em linha com a articulação. Realizamos a identificação da lesão de Bankart (-Figura 3), o desbridamento da borda anterior da glenóide e a abertura do intervalo dos rotadores até a visualização da borda lateral do processo coracóide.

2. Preparo da borda anterior da glenoide, dissecção do tendão conjunto e realização dos portais midsub e peitoral (visualização: portal anterolateral; portal de trabalho: anterior)

Neste passo, passamos a ocupar o espaço anterior do ombro. Com a utilização do portal anterolateral preparamos a borda anterior da glenoide ( - Figura 4 ), limpando todo o lábio residual e podemos visualizar o intervalo entre o tendão conjunto e o deltoide, assim como entre o tendão conjunto e o subescapular. Neste passo, identificamos os nervos axilar e musculocutâneo e liberamos as aderências laterais do tendão conjunto.

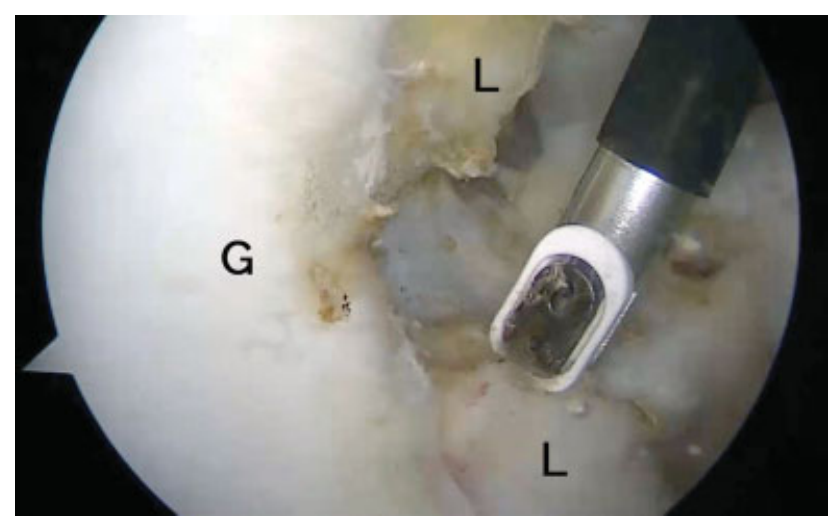

Fig. 3 Identificação da lesão labral com visualização pelo portal posterior (L) utilizando o portal posterior. (G) Glenoide. (L) Labrum. 


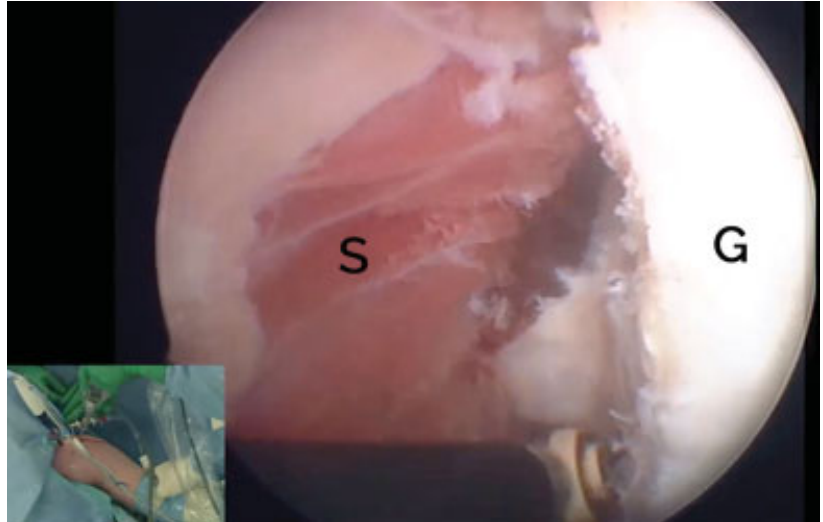

Fig. 4 Visualização da borda anterior da glenoide (G) pelo portal ântero-lateral.

O próximo passo foi a realização do portal midsub que é realizado $1,0 \mathrm{~cm}$ acima da prega axilar e em linha com 0 processo coracoide. Por meio deste portal, podemos visualizar adequadamente o processo coracoide desde sua porção lateral até sua porção medial, assim como o tendão do músculo subescapular em toda a sua extensão craniocaudal.

Neste passo, realizamos também, de dentro para fora, o portal peitoral ou "suicida" por onde será feito o split do subescapular e a passagem do enxerto com fixação na borda anterior da glenóide.

Este passo é de fundamental importância para a adequada fixação do enxerto na borda anterior da glenóide, uma vez que a colocação do mesmo muito lateral resultará em uma posição lateral do enxerto e consequente impacto com a cabeça umeral.

3. Esqueletização e Preparo do Processo coracoide (visualização: portal midsub; portais de trabalho: peitoral e coracoide)

A correta visualização do processo coracoide ( - Figura 5) é fundamental para o processo de esqueletização, liberando-o de suas inserções ligamentares e musculares.

A soltura do tendão do músculo peitoral menor é uma etapa delicada que deve ser feita cuidadosamente, pois o nervo musculocutâneo encontra-se imediatamente posterior ao mesmo. A completa liberação do tendão conjunto de

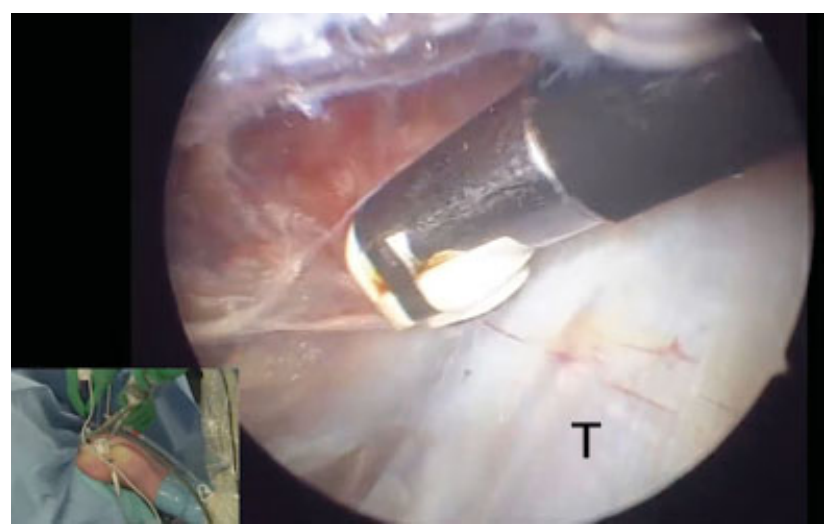

Fig. 5 Visualização do processo coracoide pelo portal midsub e esqueletização medial e lateral. (T) Tendão conjunto.

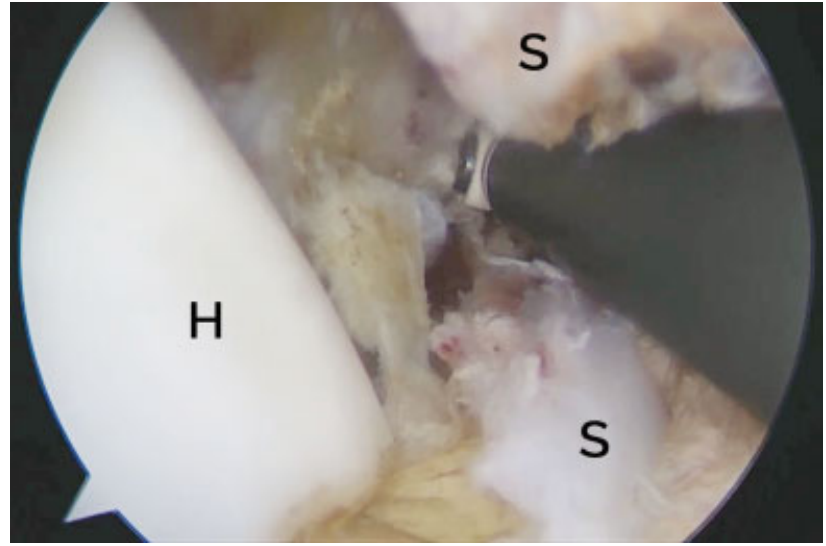

Fig. 6 Split do tendão subescapular (S) com visualização pelo portal midsub. (H) Cabeça humeral.

suas aderências e vinculas é importante para sua adequada mobilização e transferência na etapa seguinte.

Após a esqueletização do processo coracoide foram feitos os furos no processo coracoide usando guia alfa e beta (DePuy Synthes, Warsaw, IN, EUA) e a colocação de dois Top Hats deslizantes (DePuy Synthes, Warsaw, IN, EUA). Estes implantes permitem o deslizamento dos parafusos com a compressão do processo coracoide contra a borda anterior da glenoide. Após a esqueletização e preparo, o processo coracoide é osteotomizado na sua base com o uso de osteótomo.

4. Split do subescapular (visualização: portal midsub; portal de trabalho: peitoral)

O próximo passo foi o "split" do músculo subescapular (-Figura 6) que deve ser feito entre os $2 / 3$ superiores e o $1 / 3$ inferior, com a identificação do nervo axilar e a sua proteção.

5. Transferência e Fixação do Processo Coracoide (visualização: portais midsub, anterolateral e posterior; portal de trabalho: peitoral)

Com o uso de dois guias ( - Figura 7) tubulares canulados que atravessam uma cânula dupla, o processo coracoide é fixado e assim pode ser mobilizado como um joystick. Com o processo coracoide estabilizado, podemos atravessar o mesmo pelo split realizado no subescapular. Como os guias

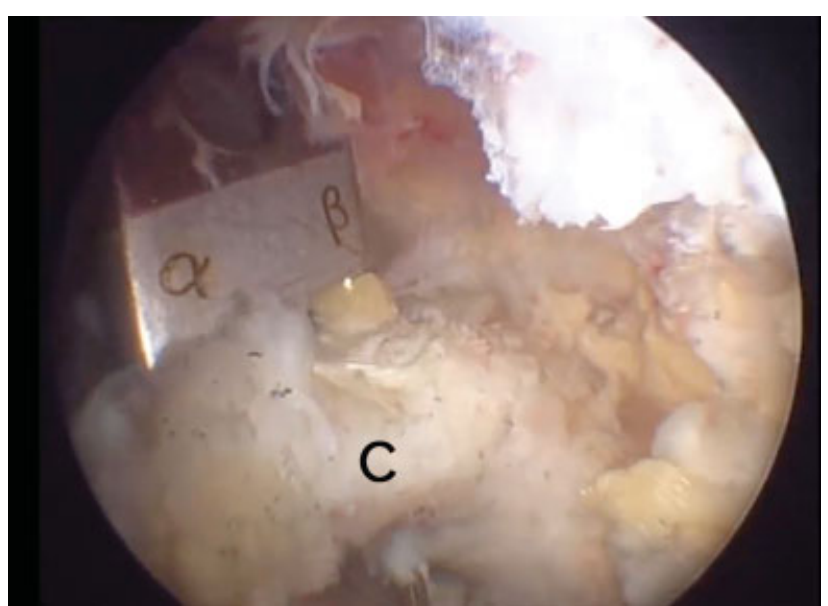

Fig. 7 Preparação do coracoide $(C)$ com visualização pelo portal Midsub. $(\alpha \beta)$ Guias alfa e beta. 


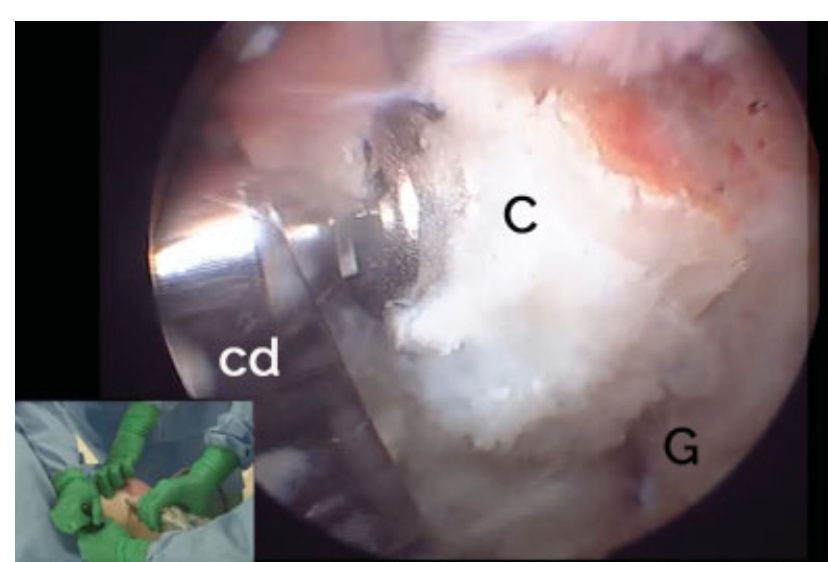

Fig. 8 Fixação do coracoide $(C)$ na borda anterior da glenoide $(G)$ com parafusos passados pela cânula dupla (cd) através de trabalho pelo portal peitoral.

são canulados, uma vez encontrada a posição adequada, o enxerto é fixado na borda anterior da glenoide com dois fios de Kirschner.

Esta etapa ainda é realizada sem um adequado guia de posicionamento na borda anterior da glenoide. Alguns parâmetros devem ser obedecidos, tais como o paralelismo entre os fios passados pelo enxerto e a posição do enxerto na borda anterior da glenoide, que deve estar abaixo da linha do equador, alinhado com a superfície articular, evitando enxertos posicionados muito mediais ou laterais.

Uma vez encontrado o adequado posicionamento do enxerto na borda anterior da glenoide, os guias canulados são retirados, e podemos proceder à brocagem e passagem dos parafusos (-Figura 8). Como a broca apresenta medida de comprimento, podemos aferir o tamanho dos parafusos a serem utilizados assim que a mesma transpassa a cortical posterior da glenoide. Na nossa experiência, o tamanho destes parafusos varia entre 32 e $36 \mathrm{~mm}$ dependendo do tamanho do paciente e do posicionamento na glenoide.

Nosso protocolo de pós-operatório inclui imobilização em tipoia e mobilidade passiva assim que permitido pela dor e mantida por até 6 semanas, quando se inicia o fortalecimento. Os pacientes são liberados para a prática de atividade física em média após 4 meses e um controle de TC para a identificação da consolidação do enxerto.

Nos 10 primeiros pacientes, o procedimento foi feito de maneira artroscópica com subsequente exposição aberta do sítio cirúrgico para checagem e ajustes necessários. Os 20 casos seguintes foram feitos de maneira totalmente artroscópica.

\section{Resultados}

O tempo de seguimento foi de no mínimo 6 meses, variando de 6 a 26 meses; não houve exclusão de nenhum paciente operado por advento de outra lesão não relacionada. No grupo estudado, $26.7 \%$ (8/30) dos pacientes eram do gênero feminino e a média de idade foi de 34 anos (de 19 a 55 anos, mínimo e máximo).

Nos 10 primeiros casos, o procedimento foi feito de maneira artroscópica com subsequente exposição aberta do sítio cirúrgico previamente planejada para checagem e ajustes necessários, sendo que não houve conversão dos casos para a realização do procedimento em si, mas sim para checagem e garantia de conformidade dos reparos. As principais dificuldades técnicas observadas e corrigidas nos primeiros casos foram: (1) split muito superior do subescapular; (2) perfuração e preparo do coracoide não alinhado com o eixo do mesmo; (3) posicionamento lateral do enxerto.

Estas dificuldades foram encontradas principalmente pelo posicionamento inadequado dos portais. 0 portal midsub permite a visualização do tendão do subescapular em toda a sua extensão com a realização do split na altura mais adequada. $\mathrm{O}$ portal peitoral (também denominado de portal suicida) realizado em linha com a articulação é fundamental para o adequado posicionamento do enxerto na borda anterior da glenoide. Nos primeiros casos da nossa curva de aprendizado, por receio da realização destes dois portais, estes dois passos da cirurgia poderiam ter sido dificultados. A partir do $11^{\circ}$ caso, passamos a realizar o procedimento totalmente por via artroscópica, e aí sim, puderam-se observar algumas complicações inerentes à curva de aprendizado. As complicações aconteceram nos casos de número $6,11,12,14$, e 20 . Vale ressaltar que do $21^{\circ}$ ao $30^{\circ}$ casos não foram observadas complicações.

O número total de complicações foi de 5 eventos $(16,7 \%)$, sendo 1 intra-operatória (3.3\%), 2 eventos pós-operatórios transitórios (6,7\%) e 2 eventos pós-operatórios que levaram a um novo procedimento cirúrgico de forma eletiva $(6,7 \%)$. Nenhum paciente teve mais de uma complicação, totalizando 5 casos (16,7\%). Nenhum caso teve sangramento inesperado no ato cirúrgico, tampouco houve hematoma observado no pósoperatório. As suturas evoluíram como o esperado e nenhuma deiscência ocorreu. Nenhuma infecção foi observada, sendo profunda ou de ferida operatória.

Em 1 caso (3,3\%) ocorreu fratura do processo coracoide no momento de fixação do enxerto na borda anterior da glenoide e optamos por converter a artroscopia para cirurgia aberta. $\mathrm{O}$ fragmento residual foi suficiente para manter um parafuso fixando o enxerto. Por motivo de segurança, optamos pela conversão para cirurgia aberta para checagem e finalização do procedimento. Este caso foi o $20^{\circ}$ caso da série. Dois casos (6,7\%) apresentaram neuropraxia do músculo cutâneo, que foi revertida com o período de fisioterapia, sem sequelas e nem necessidade de medicações. Dois pacientes (6,7\%) evoluíram com limitação da rotação externa por posicionamento muito lateral dos parafusos. Para tais casos houve necessidade de nova intervenção para retirada do material de síntese e "emagrecimento" da borda lateral do enxerto para liberação articular. Os casos evoluíram de maneira satisfatória após tal procedimento.

Não houve caso de recidiva na amostra durante o tempo de seguimento estudado. Vale lembrar que um seguimento maior pode trazer os resultados definitivos deste procedimento.

\section{Discussão}

É consenso na literatura que a cirurgia de Bristow-Latarjet é ferramenta útil dentro do arsenal do tratamento cirúrgico das instabilidades glenoumerais, ocupando papel importante no 
tratamento dos pacientes com lesões ósseas, lesões Hill-Sachs do tipo "engaging”, lesões HAGL, em pacientes jovens e envolvidos em esportes de contato ou nas recidivas das lesões de Bankart. ${ }^{19,20}$ Os estudos mostram bons resultados apesar de incidência de complicações que preocupam tanto em curto, médio ou longo prazo.

As principais complicações são sangramento, neuroapraxias do musculocutâneo e axilar, problemas relacionados ao enxerto (fraturas ou solturas de material de síntese) e a excessiva limitação da rotação externa que pode levar a artrose. $^{10,11}$ Baseado na literatura recente, a cirurgia de Latarjet, quando realizada por via artroscópica, tem um risco de lesões nervosas entre 0 e 1,6\%, significativamente menor que a cirurgia aberta. Acreditamos que isso ocorre pela direta visualização das estruturas neurovasculares, menor necessidade de afastamento, além de menor tração direta sobre o enxerto, uma vez que o mesmo não é tracionado para fora da articulação para o seu preparo. 0 estudo de Zhu et al $^{14}$ confirma que uma indicação para o Latarjet artroscópico é o seu menor risco de lesões nervosas ou vasculares.

Outro ponto importante é o posicionamento do enxerto: quando colocado muito lateral pode levar à excessiva restrição da rotação externa e artrose, enquanto o posicionamento muito alto ou medial pode levar a altas taxas de recidiva. A literatura mostra que o mau posicionamento do enxerto varia entre 20 e $67 \%$ na cirurgia aberta e entre 4 e $24 \%$ quando a cirurgia é realizada por via artroscópica. ${ }^{21-23}$ Acreditamos que a visão aumentada proporcionada pelo artroscópio aliado à possibilidade de olharmos por diversos ângulos faz com que o posicionamento seja mais preciso na técnica artroscópica.

No nosso entendimento, o cirurgião e equipe devem estar treinados para realizar a cirurgia por via aberta, ter conhecimento e familiaridade com o instrumental artroscópico, e a cirurgia pode e deve ser convertida para aberta a qualquer sinal de perigo para o paciente ou dificuldade técnica que ponha em risco a precisão e o resultado final. Para qualquer procedimento, a transição da cirurgia aberta para a artroscopia é um processo com uma curva de aprendizado e um aprimoramento contínuo. A curva de aprendizado do Latarjet artroscópico tem sido demonstrada na literatura, mas também existe para o Latarjet aberto. Autores ${ }^{15,24,25}$ relatam que entre 18 a 30 casos são necessários para o cirurgião e a equipe sentirem segurança no procedimento, e foi bastante semelhante com a nossa experiência, na qual a checagem sistemática por via aberta foi realizada nos 10 primeiros casos com alguns ajustes necessários, seguindo por 20 casos totalmente artroscópicos com cinco complicações nos 10 primeiros casos e nenhuma complicação nos 10 casos finais da série. A técnica artroscópica demanda certa experiência prévia com artroscopia, mas foi possível obter um número baixo de complicações mesmo em uma experiência inicial.

Particularmente nesta cirurgia, o trabalho em equipe é fundamental. Um conhecimento da técnica por parte de toda a equipe, assim como um adequado controle dos parâmetros de pressão e fluxo por parte do anestesista é fundamental para não aumentar excessivamente o tempo cirúrgico e para o sucesso do procedimento. Com a evolução da técnica e instrumentais, as complicações tendem a se minimizar, como mostram os estudos recentes, e podemos ter uma técnica bastante eficaz para a estabilização da articulação glenoumeral com pequenas taxas de complicações. Aliado a isso, os novos estudos que estão sendo realizados avaliando posicionamento do enxerto, ângulo de fixação dos parafusos e análise da reabsorção do enxerto só trazem melhorias e um melhor entendimento da técnica e do que acontece com nossos pacientes no longo prazo.

Dentre as principais vantagens da técnica artroscópica, podemos citar o mais preciso posicionamento do enxerto junto à borda anterior da glenoide, a adequada visualização das estruturas neurovasculares e o tratamento das lesões associadas, além das vantagens inerentes às cirurgias minimamente invasivas (menos dor no pós-operatório, menor taxa de infecção e mais rápida reabilitação, além de ser esteticamente mais aceitável). ${ }^{8,9}$

Assim como em outros artigos da literatura contemporâneos, ${ }^{13}$ este estudo apresentou resultados de curto a médio prazo de um grupo de pacientes tratados através de Latarjet artroscópico por grupo de cirurgiões em início de curva de aprendizado. Estudos subsequentes devem mostrar resultados de longo prazo sobre a aplicação de Latarjet por via artroscópica para correção de luxação anterior do ombro com perda óssea glenoidal.

\section{Conclusão}

Os resultados preliminares encontrados no presente estudo com o uso da técnica de Latarjet artroscópico no tratamento da instabilidade anterior do ombro são animadores. A incidência de complicações foi baixa em relação a lesões de estruturas neurovasculares no posicionamento do enxerto.

Conflito de Interesses

Os autores declaram não haver conflito de interesses.

\section{Agradecimentos}

Os autores agradecem a Antonio Guilherme Garofo, que contribuiu a este trabalho com méritos de coautor, mas não pôde integrar a lista de autoria por conta de diretriz de publicação. Ainda, os autores agradecem ao Bruno Rodrigo Crispim Lima pela brilhante colaboração gráfica com ilustração da - Figura 1.

\section{Referências}

1 D'Angelo D. Concept of healing of recurrent shoulder dislocation. Rev Bras Ortop 2014;49(04):420-425

2 Bankart AS. Recurrent or habitual dislocation of the shoulderjoint. BMJ 1923;2(3285):1132-1133

3 Latarjet M. [Treatment of recurrent dislocation of the shoulder]. Lyon Chir 1954;49(08):994-997

4 Helfet AJ. Coracoid transplantation for recurring dislocation of the shoulder. J Bone Joint Surg Br 1958;40-B(02):198-202

5 Ferreira Filho AA. Tratamento da luxacao anterior recidivante do ombro pela tecnica de bristow - latarjet [tese]. São Paulo: Faculdade de Medicina da Universidade de São Paulo; 1984

6 Moura DL, Reis ARE, Ferreira J, Capelão M, Cardoso JB. Modified Bristow-Latarjet procedure for treatment of recurrent traumatic 
anterior glenohumeral dislocation. Rev Bras Ortop 2018;53(02): 176-183

7 Hovelius L, Sandström B, Sundgren K, Saebö M. One hundred eighteen Bristow-Latarjet repairs for recurrent anterior dislocation of the shoulder prospectively followed for fifteen years: study I-clinical results. J Shoulder Elbow Surg 2004;13(05): 509-516

8 Karlsson J, Magnusson L, Ejerhed L, Hultenheim I, Lundin O, Kartus J. Comparison of open and arthroscopic stabilization for recurrent shoulder dislocation in patients with a Bankart lesion. Am J Sports Med 2001;29(05):538-542

9 Freedman KB, Smith AP, Romeo AA, Cole BJ, Bach BR Jr. Open Bankart repair versus arthroscopic repair with transglenoid sutures or bioabsorbable tacks for Recurrent Anterior instability of the shoulder: a meta-analysis. Am J Sports Med 2004;32(06): $1520-1527$

10 Lafosse L, Lejeune E, Bouchard A, Kakuda C, Gobezie R, Kochhar T. The arthroscopic Latarjet procedure for the treatment of anterior shoulder instability. Arthroscopy 2007;23(11):1242. e1-1242.e5

11 Lafosse L, Boyle S, Gutierrez-Aramberri M, Shah A, Meller R. Arthroscopic latarjet procedure. Orthop Clin North Am 2010;41 (03):393-405

12 Dumont GD, Fogerty S, Rosso C, Lafosse L. The arthroscopic latarjet procedure for anterior shoulder instability: 5-year minimum follow-up. Am J Sports Med 2014;42(11):2560-2566

13 Athwal GS, Meislin R, Getz C, Weinstein D, Favorito P. Short-term Complications of the Arthroscopic Latarjet Procedure: A North American Experience. Arthroscopy 2016;32(10):1965-1970

14 Zhu YM, Jiang C, Song G, Lu Y, Li F. Arthroscopic Latarjet Procedure With Anterior Capsular Reconstruction: Clinical Outcome and Radiologic Evaluation With a Minimum 2-Year Follow-Up. Arthroscopy 2017;33(12):2128-2135

15 Castricini R, De Benedetto M, Orlando N, Rocchi M, Zini R, Pirani P. Arthroscopic Latarjet procedure: analysis of the learning curve. Musculoskelet Surg 2013;97(Suppl 1):93-98
16 Schulze-Borges J, Agneskirchner JD, Bobrowitsch E, et al. Biomechanical comparison of open and arthroscopic Latarjet procedures. Arthroscopy 2013;29(04):630-637

17 Boileau P, Saliken D. Editorial Commentary: The Wake of the Dragon: Will the Orthopaedic Community Adopt the Shoulder Arthroscopic Latarjet Procedure as We Adopted the Arthroscopic Rotator Cuff Repair? Arthroscopy 2017;33(12):2139-2143

18 Balg F, Boileau P. The instability severity index score. A simple preoperative score to select patients for arthroscopic or open shoulder stabilisation. J Bone Joint Surg Br 2007;89(11):1470-1477

19 da Silva LA, da Costa Lima ÁG, Kautsky RM, Santos PD, do Val Sella $G$, Checchia SL. Evaluation of the results and complications of the Latarjet procedure for recurrent anterior dislocation of the shoulder. Rev Bras Ortop 2015;50(06):652-659

20 Ikemoto RY, Murachovisky J, Nascimento LG, et al. Results from latarjet surgery for treating traumatic anterior shoulder instability associated with bone erosion in the glenoid cavity, after minimum follow-up of one year. Rev Bras Ortop 2015;46(05):553-560

21 Kraus TM, Martetschläger F, Graveleau N, et al. CT-based quantitative assessment of the surface size and en-face position of the coracoid block post-Latarjet procedure. Arch Orthop Trauma Surg 2013;133(11):1543-1548

22 Casabianca L, Gerometta A, Massein A, et al. Graft position and fusion rate following arthroscopic Latarjet. Knee Surg Sports Traumatol Arthrosc 2016;24(02):507-512

23 Boileau P, Thélu CÉ, Mercier N, et al. Arthroscopic Bristow-Latarjet combined with bankart repair restores shoulder stability in patients with glenoid bone loss. Clin Orthop Relat Res 2014;472 (08):2413-2424

24 Kany J, Flamand O, Grimberg J, et al. Arthroscopic Latarjet procedure: is optimal positioning of the bone block and screws possible? A prospective computed tomography scan analysis. J Shoulder Elbow Surg 2016;25(01):69-77

25 Cunningham G, Benchouk S, Kherad O, Lädermann A. Comparison of arthroscopic and open Latarjet with a learning curve analysis. Knee Surg Sports Traumatol Arthrosc 2016;24(02):540-545 